\title{
Aspectos epidemiológicos da tuberculose no Município de Caxias, do Estado do
}

\section{Maranhão}

\author{
Epidemiological aspects of tuberculosis in the City of Caxias, in the State of Maranhão \\ Aspectos epidemiológicos de la tuberculosis en la Ciudad de Caxias, en el Estado de Maranhão
}

Recebido: 24/02/2021 | Revisado: 07/03/2021 | Aceito: 13/03/2021 | Publicado: 20/03/2021

Rogério Cruz Mendes

ORCID: https://orcid.org/0000-0002-9339-6133

Centro Universitário de Ciências e Tecnologia do Maranhão, Brasil

E-mail: rogeriocruz82@yahoo.com

Wenderson Costa da Silva

ORCID: https://orcid.org/0000-0001-6031-9775

Universidade Estadual do Maranhão, Brasil

E-mail: wendersoncosta09@hotmail.com

Chrisllayne Oliveira da Silva

ORCID: https://orcid.org/0000-0002-0844-0268

Centro Universitário de Ciências e Tecnologia do Maranhão, Brasil E-mail: chris-layne10@hotmail.com

Alanna Nunes Soares

ORCID: https://orcid.org/0000-0002-0904-4515

Centro Universitário de Ciências e Tecnologia do Maranhão, Brasil

E-mail: alanna_ns@hotmail.com

Karine Costa Melo

ORCID: https://orcid.org/0000-0001-8253-859X

Centro Universitário de Ciências e Tecnologia do Maranhão, Brasil

E-mail: karinemelo09@gmail.com

Deusilene dos Santos Anjos

ORCID: https://orcid.org/0000-0001-7612-987X

Centro Universitário Planalto do Distrito Federal, Brasil

E-mail: Deusilene.cx@gmail.com

Ianeska Bárbara Ribeiro do Nascimento

ORCID: https://orcid.org/0000-0002-5319-289X

Centro Universitário de Ciências e Tecnologia do Maranhão, Brasil

E-mail: ianeska@outlook.com

Linccon Fricks Hernandes

ORCID: https://orcid.org/0000-0002-7642-3080

Escola Superior de Ciências da Santa Casa de Misericórdia de Vitória, Brasil

E-mail: fricksjr@hotmail.com

Layse Siqueira Costa Miranda

ORCID: https://orcid.org/0000-0001-5942-4666

Centro Universitário de Ciências e Tecnologia do Maranhão, Brasil

E-mail: layse62@gmail.com

Brunna Matos Sousa

ORCID: https://orcid.org/0000-0002-7009-6488

Centro Universitário de Ciências e Tecnologia do Maranhão, Brasil

E-mail:brunnamattos4@gmail.com

Lia Marinho da Silva Queiroz

ORCID: https://orcid.org/0000-0002-5437-6877

Centro Universitário de Ciências e Tecnologia do Maranhão, Brasil

E-mail: liamarinho1998@gmail.com

Lorena da Conceição Sousa Silva

ORCID: https://orcid.org/0000-0003-0360-3387

Centro Universitário de Ciências e Tecnologia do Maranhão, Brasil

E-mail: lorenasousasilva@outlook.com

Kaio Germano Sousa da Silva

ORCID: https://orcid.org/0000-0003-4236-6230

Centro Universitário de Ciências e Tecnologia do Maranhão, Brasil

E-mail: kaiogsds@hotmail.com

Rodrigo de Lima Souza

ORCID: https://orcid.org/0000-0002-0079-8919

Centro Universitário de Ciências e Tecnologia do Maranhão, Brasil

E-mail: rodrigosousa_caxias@ hotmail.com 


\author{
Lucas Sousa Guimarães \\ ORCID: https://orcid.org/0000-0002-9228-8114 \\ Centro Universitário de Ciências e Tecnologia do Maranhão, Brasil \\ E-mail: lucasguimaraes0310@gmail.com \\ Ludmylla Lima da Conceição \\ ORCID: https://orcid.org/0000-0001-8732-4304 \\ Centro Universitário de Ciências e Tecnologia do Maranhão, Brasil \\ E-mail: ludlimamylla@outlook.com
}

\begin{abstract}
Resumo
A Tuberculose (TB) é uma doença infectocontagiosa e endêmica, provocada pelo Mycobacterium tuberculosis, chegando a atingir quase todos os órgãos e tecidos do corpo humano. Este estudo teve como objetivo descrever os aspectos epidemiológicos da tuberculose no município de Caxias do Estado do Maranhão no período de 2015-2019. Trata-se de estudo documental, descritivo, retrospectivo, com uma abordagem quantitativa dos dados, realizado no período de 2015 a 2019, utilizando dados do Sistema de Informação de Agravos de Notificação (SINAN) do Ministério da Saúde. Este estudo avaliou 325 casos de TB no período citado. Pode observar que houve prevalência de casos de TB no sexo masculino (67,7\%), em que houve predominância da faixa etária de 20 a 39 anos (33,5\%). Com relação a raça, houve prevalência estatisticamente significativa da parda (70,8\%), em que a maior parte dos indivíduos apresentavam baixa escolaridade. Com relação a prevalência, o ano de 2016 apresentou maiores índices (23,7\%). A forma clinica predominante da doença foi a pulmonar $(91,4 \%)$, em que quase totalidade eram casos novos $(82,8 \%)$. Diante dos problemas encontrados torna-se claro que a TB continua sendo um grande problema de saúde pública. É necessário que mais ações voltadas ao combate da patologia sejam colocadas em prática e que os governos enxerguem a extensão do problema. Considera-se que mais estudos devem ser realizados nessa linha de investigação, a fim de provar a necessidade de buscar novas formas de abordar a temática, visando diminuir o número de casos.
\end{abstract}

Palavras-chave: Prevalência; Epidemiologia; Enfermagem.

\begin{abstract}
Tuberculosis (TB) is an infectious and endemic infectious disease, caused by Mycobacterium tuberculosis, reaching almost all organs and tissues of the human body. This study aimed to describe the epidemiological aspects of tuberculosis in the city of Caxias in the state of Maranhão in the period 2015-2019. This is a documentary, descriptive, retrospective study, with a quantitative approach to the data, carried out from 2015 to 2019, using data from the Ministry of Health's Notifiable Diseases Information System (SINAN). This study evaluated 325 cases of TB in the mentioned period. It can be observed that there was a prevalence of TB cases in males $(67.7 \%)$, in which there was a predominance of the age group of 20 to 39 years (33.5\%). Regarding race, there was a statistically significant prevalence of brown skin $(70.8 \%)$, in which most individuals had low education. With regard to prevalence, 2016 had the highest rates $(23.7 \%)$. The predominant clinical form of the disease was pulmonary $(91.4 \%)$, in which almost all were new cases $(82.8 \%)$. In view of the problems encountered, it is clear that TB remains a major public health problem. It is necessary that more actions aimed at combating pathology be put into practice and that governments see the extent of the problem. It is considered that more studies should be carried out in this line of investigation, in order to prove the need to search for new ways to approach the theme, aiming to decrease the number of cases.
\end{abstract}

Keywords: Prevalence; Epidemiology; Nursing.

\title{
Resumen
}

La tuberculosis (TB) es una enfermedad infecciosa infecciosa y endémica, causada por Mycobacterium tuberculosis, que llega a casi todos los órganos y tejidos del cuerpo humano. Este estudio tuvo como objetivo describir los aspectos epidemiológicos de la tuberculosis en la ciudad de Caxias en el estado de Maranhão en el período 2015-2019. Se trata de un estudio documental, descriptivo, retrospectivo, con abordaje cuantitativo de los datos, realizado de 2015 a 2019 , con datos del Sistema de Información de Enfermedades Notificables del Ministerio de Salud (SINAN), que evaluó 325 casos de TB en el mencionado período. Se puede observar que hubo una prevalencia de casos de TB en el sexo masculino (67,7\%), en el que hubo predominio del grupo etario de 20 a 39 años (33,5\%). En cuanto a la raza, hubo una prevalencia estadísticamente significativa de piel morena (70,8\%), en la que la mayoría de los individuos tenía baja escolaridad. En cuanto a la prevalencia, 2016 tuvo las tasas más altas (23,7\%). La forma clínica predominante de la enfermedad fue la pulmonar $(91,4 \%)$, en la que casi todos fueron casos nuevos $(82,8 \%)$. En vista de los problemas encontrados, está claro que la tuberculosis sigue siendo un problema importante de salud pública. Es necesario que se pongan en práctica más acciones encaminadas a combatir la patología y que los gobiernos vean la magnitud del problema. Se considera que se deben realizar más estudios en esta línea de investigación, con el fin de demostrar la necesidad de buscar nuevas formas de abordar el tema, con el objetivo de disminuir el número de casos.

Palabras clave: Prevalencia; Epidemiología; Enfermería. 


\section{Introdução}

A Tuberculose (TB) é uma doença infectocontagiosa e endêmica, provocada pelo Mycobacterium tuberculosis, chegando a atingir quase todos os órgãos e tecidos do corpo humano. Diante do cenário mundial, a TB ainda é considerada uma das principais causas de morbidade e mortalidade. Segundo a Organização Mundial da Saúde (OMS) calcula-se que, em 2016, ocorreu 10,4 milhões de novos casos de TB (Carvalho, Cardoso, Martire, Migliori, \& Sant'Anna, 2018).

Os países não desenvolvidos são onde ocorrem as maiores manifestações de casos de TB devido as aglomerações na urbanização sem controle e consequentemente gerando o surgimento de favelas num convívio social de pobreza. Desta forma os habitantes que ali reside não possui água tratada, tratamento de esgoto e serviços de promoção e prevenção em saúde, expondo a população aos riscos de doenças infectocontagiosas e parasitárias (Carvalho, 2019).

O Brasil apesar dos avanços na área da saúde direcionado a prevenção e ao tratamento da TB, nosso país ainda consta na lista desses países que possui inúmeros casos. Principalmente nas regiões periféricas dos centros urbanos e por incrível que pareça também nas zonas rurais por possui uma população desprovida de água tratada e acesso fácil aos serviços de saúde (Soares, Nunes, Leite, Soares, \& Medeiros, 2019).

Mesmo perante a tantas tecnologias desenvolvidas na busca por soluções, especialmente aquelas que abrangem ao diagnóstico, ao tratamento e aos acessos as medidas de prevenção da TB. Ainda existem os fatores desencadeantes como os determinantes sociais relacionados à saúde e a degradação do meio ambiente que infelizmente são condições cruciais para o aumento dos casos (Ferreira Neto, Oliveira, \& Pimenta, 2020).

Os profissionais de enfermagem possuem uma importância fundamental na prevenção e tratamento da TB, pois é o profissional de saúde que tem maior convívio com o paciente, familiares e população. Fornecendo continuamente orientações quanto ao não abandono e as intercorrências na busca de tratar a infecção instalada, focando sempre na promoção em saúde e estilo de vida saudável dos indivíduos (Santana, Fraga, \& Moreira, 2018).

Assim, diante da relevância do assunto em questão, observou-se que a TB ainda é muito prevalente na sociedade brasileira e no Maranhão a incidência atinge números consideravelmente preocupantes. Isso despertou a descobrir os números reais dos aspectos epidemiológicos da TB na cidade de Caxias-MA.

Estudos demonstraram que mesmo perante as diversas formas de tecnologias da informação, as pessoas ainda não são conhecedoras e nem capazes de desenvolverem cuidados de prevenção como prática de higiene pessoal diária, higiene domiciliar, boas práticas alimentares, atividade física e de laser. E também evitar ações prejudiciais como o uso excessivo do tabagismo e etilismo que afetam o estado de saúde e oportunizam com maior facilidade para o aparecimento da TB.

Este estudo almeja despertar na população, principalmente a classe social de baixa renda que infelizmente é a mais afetada pela TB e que tal doença mesmo em pleno século XXI, ainda surge rotineiramente no convívio em sociedade. Assim sendo, os profissionais de saúde em especial aqueles atuantes na Atenção Primária possuem um papel fundamental para o acompanhamento e tratamento de pacientes infectados e, nas realizações de ações que visam prevenir o aparecimento da TB.

Diante do exposto, este estudo teve como questão norteadora: Quais os aspectos epidemiológicos da tuberculose no município de Caxias, do Estado do Maranhão? Para tal, o objetivo geral deste estudo foi descrever os aspectos epidemiológicos da tuberculose no município de Caxias do Estado do Maranhão no período de 2015-2019. E especificamente: determinar a prevalência de TB no período citado; levantar dados sociodemográficos dos casos de TB; identificar os casos de TB de acordo com a forma clínica da doença, tipo de entrada e a evolução dos casos; e analisar a presença de doenças e agravos associados.

\section{Metodologia}

Trata-se de estudo documental, descritivo, retrospectivo, com uma abordagem quantitativa dos dados, realizado no período de 2015 a 2019, utilizando dados do SINAN do Ministério da Saúde. Os dados foram coletados a partir das 
informações disponíveis no site do DATASUS. No Portal, os dados foram obtidos acessando o acompanhamento dos dados de TB no maranhão, restringindo os dados ao município de Caxias-MA. A coleta de dados ocorreu em novembro de 2020.

Os estudos documentais apresentam semelhança aos estudos bibliográficos, pois consistem na utilização de dados ou materiais já existente, porém nos estudos documentais esses dados ainda não foram totalmente explorados e analisados, possibilitando que o pesquisador, tenha uma fonte de dados direta e realizando uma análise precisa. Estudos retrospectivos é uma modalidade de pesquisa realizada a partir da existência prévia e registros dos dados (Carvalho, Duarte, Menezes \& Souza, 2019).

A abordagem quantitativa ocorre por meio da coleta de dados numéricos, que pode ser por meio de medições de grandezas, utilizando os algarismos numéricos, que permitirá o agrupamento e análise dos dados conforme o pesquisador determine, ou seja, através de estatística, porcentagem, probabilidade, fórmulas matemáticas dentre outras (Pereira et al., 2018).

O estudo analisou os aspectos epidemiológicos dos casos de TB notificados no município de Caxias-MA. A amostra do estudo foi composta por todos os casos de TB notificados no período de 2015 a 2019. Foram analisados 325 casos. Os critérios de inclusão foram todos os casos de TB notificados no município supracitado, no período de 2015 a 2019, constantes da base de dados DATASUS do Ministério da Saúde, inseridos no programa SINAN. Os critérios de exclusão foram os casos que foram notificados fora do município citado, que não constem do SINAN, além daqueles fora do recorte temporal estabelecido.

Os dados do estudo foram obtidos através da identificação e correlação de variáveis, tais como: faixa etária, sexo, raça, escolaridade, forma clínica da TB, modo de entrada, evolução dos casos, doenças e agravos associados. Os dados foram organizados e tabulados utilizando-se o Microsoft Excel versão 2016 para Windows. Foi realizada uma análise descritiva, e os resultados foram apresentados por meio de frequência simples e absoluta e dispostos em tabelas e gráficos.

Pelo fato do estudo não envolver diretamente pesquisa com seres humanos e a base dados do SINAN ser de acesso público, não contemplando dessa forma as normas estabelecidas pela Resolução Conselho Nacional de Saúde (CNS) $n^{\circ}$ 466/2012 e suas complementares, não houve a necessidade do projeto ser enviado à Plataforma Brasil para a análise de um Comitê de Ética em Pesquisa (CEP).

\section{Resultados e Discussão}

Este estudo avaliou 325 casos de TB no período de 2015 a 2019 no município de Caxias do estado do Maranhão. Quanto as variáveis sociodemográficas em todo período analisado, pode observar que houve prevalência de casos no sexo masculino, com 67,7\% (220), em que houve predominância da faixa etária de 20 a 39 anos (33,5\%), seguido de 40 a 59 anos $(33,2 \%)$. Contudo, houve um grande percentual de idosos na faixa etária de 60 a 80 anos ou mais $(25,8 \%)$. Com relação a raça, houve prevalência estatisticamente significativa da parda, com 70,8\% dos casos. E se tratando da escolaridade os indivíduos com ensino fundamental incompleto e os analfabetos tiveram maior percentual de ocorrência, com 36,3\% e $27,1 \%$, respectivamente, conforme demonstrado na Tabela 1. 
Tabela 1. Distribuição dos dados sociodemográficos dos casos de Tuberculose no período de 2015 a 2019 no município de Caxias, MA. Caxias, MA, Brasil, 2020.

\begin{tabular}{|c|c|c|c|c|c|c|c|c|c|c|c|c|}
\hline \multicolumn{13}{|c|}{ Ano } \\
\hline \multirow[t]{2}{*}{ Variáveis } & \multicolumn{2}{|c|}{2015} & \multicolumn{2}{|c|}{2016} & \multicolumn{2}{|c|}{2017} & \multicolumn{2}{|c|}{2018} & \multicolumn{2}{|c|}{2019} & \multicolumn{2}{|c|}{ Total } \\
\hline & $\mathbf{N}$ & $\%$ & $\mathbf{N}$ & $\%$ & $\mathbf{N}$ & $\%$ & $\mathbf{N}$ & $\%$ & $\mathbf{N}$ & $\%$ & $\mathbf{N}$ & $\%$ \\
\hline \multicolumn{13}{|l|}{ Sexo } \\
\hline Masculino & 40 & 12,3 & 48 & 14,8 & 43 & 13,2 & 44 & 13,5 & 45 & 13,8 & 220 & 67,7 \\
\hline Feminino & 13 & 4,0 & 29 & 8,9 & 18 & 5,5 & 23 & 7,1 & 22 & 6,8 & 105 & 32,3 \\
\hline Total & 53 & 16,3 & 77 & 23,7 & 61 & 18,8 & 67 & 20,6 & 67 & 20,6 & 325 & 100,0 \\
\hline \multicolumn{13}{|l|}{ Faixa etária } \\
\hline$<1$ ano & - & - & - & - & 1 & 0,3 & - & - & - & - & 1 & 0,3 \\
\hline 5 a 9 anos & - & - & - & - & - & - & - & - & 1 & 0,3 & 1 & 0,3 \\
\hline 10 a 14 anos & 1 & 0,3 & - & - & - & - & 4 & 1,2 & 1 & 0,3 & 6 & 1,8 \\
\hline 15 a 19 anos & 4 & 1,2 & 4 & 1,2 & 1 & 0,3 & 4 & 1,2 & 3 & 0,9 & 16 & 4,9 \\
\hline 20 a 39 anos & 20 & 6,2 & 24 & 7,4 & 22 & 6,8 & 22 & 6,8 & 21 & 6,5 & 109 & 33,5 \\
\hline 40 a 59 anos & 18 & 5,5 & 25 & 7,7 & 17 & 5,2 & 25 & 7,7 & 23 & 7,1 & 108 & 33,2 \\
\hline 60 a 64 anos & 2 & 0,6 & 9 & 2,8 & 7 & 2,2 & 4 & 1,2 & 2 & 0,6 & 24 & 7,4 \\
\hline 65 a 69 anos & 1 & 0,3 & 3 & 0,9 & 4 & 1,2 & 1 & 0,3 & 4 & 1,2 & 13 & 4,0 \\
\hline 70 a 79 anos & 6 & 1,8 & 8 & 2,5 & 6 & 1,8 & 3 & 0,9 & 10 & 3,1 & 33 & 10,2 \\
\hline $80+$ & 1 & 0,3 & 4 & 1,2 & 3 & 0,9 & 4 & 1,2 & 2 & 0,6 & 14 & 4,3 \\
\hline Total & 53 & 16,3 & 77 & 23,7 & 61 & 18,8 & 67 & 20,6 & 67 & 20,6 & 325 & 100,0 \\
\hline \multicolumn{13}{|l|}{ Raça } \\
\hline Branca & 5 & 1,5 & 12 & 3,7 & 6 & 1,8 & 9 & 2,8 & 9 & 2,8 & 41 & 12,6 \\
\hline Preta & 6 & 1,8 & 8 & 2,5 & 11 & 3,4 & 16 & 4,9 & 13 & 4,0 & 54 & 16,6 \\
\hline Parda & 42 & 12,9 & 57 & 17,5 & 44 & 13,5 & 42 & 12,9 & 45 & 13,8 & 230 & 70,8 \\
\hline Total & 53 & 16,3 & 77 & 23,7 & 61 & 18,8 & 67 & 20,6 & 67 & 20,6 & 325 & 100,0 \\
\hline \multicolumn{13}{|l|}{ Escolaridade } \\
\hline Analfabeto & 12 & 3,7 & 21 & 6,5 & 18 & 5,5 & 16 & 4,9 & 21 & 6,5 & 88 & 27,1 \\
\hline $\begin{array}{l}\text { Fundamental } \\
\text { incompleto }\end{array}$ & 21 & 6,5 & 28 & 8,6 & 19 & 5,8 & 19 & 5,8 & 31 & 9,5 & 118 & 36,3 \\
\hline $\begin{array}{l}\text { Fundamental } \\
\text { completo }\end{array}$ & 2 & 0,6 & 4 & 1,2 & 3 & 0,9 & 3 & 0,9 & 4 & 1,2 & 16 & 4,9 \\
\hline $\begin{array}{l}\text { Médio } \\
\text { incompleto }\end{array}$ & 5 & 1,5 & 7 & 2,2 & 5 & 1,5 & 6 & 1,8 & 1 & 0,3 & 24 & 7,4 \\
\hline $\begin{array}{l}\text { Médio } \\
\text { completo }\end{array}$ & 7 & 2,2 & 11 & 3,4 & 9 & 2,8 & 16 & 4,9 & 8 & 2,5 & 51 & 15,7 \\
\hline $\begin{array}{l}\text { Superior } \\
\text { incompleto }\end{array}$ & 2 & 0,6 & - & - & - & - & 1 & 0,3 & - & - & 3 & 0,9 \\
\hline $\begin{array}{l}\text { Superior } \\
\text { completo }\end{array}$ & 2 & 0,6 & 3 & 0,9 & 1 & 0,3 & 1 & 0,3 & 2 & 0,6 & 9 & 0,9 \\
\hline Não se aplica & - & - & - & - & 1 & 0,3 & - & - & - & - & 1 & 0,3 \\
\hline Ignorado & 2 & 0,6 & 3 & 0,9 & 5 & 1,5 & 5 & 1,5 & - & - & 15 & 4,6 \\
\hline Total & 53 & 16,3 & 77 & 23,7 & 61 & 18,8 & 67 & 20,6 & 67 & 20,6 & 325 & 100,0 \\
\hline
\end{tabular}

Legenda: $\mathrm{N}=$ número; $\%$ = percentual.

Fonte: Ministério da Saúde/ Sistema de Informação de Agravos de Notificação (SINAN Net -2020).

Ao se traçar o perfil dos indivíduos mais acometidos pela TB, torna-se claro que a maioria é do sexo masculino. O estudo de Pinto et al. (2017) realizado no município de São Paulo também encontrou resultado semelhante, onde houve uma maior incidência de TB nesse gênero. Tal fato é explicado pela grande exposição masculina às patologias infectocontagiosas devido ao tabagismo e ao alcoolismo e também pela carência de uso dos serviços de saúde e utilização de medidas preventivas por esse público (Fontes et al., 2019; Santos \& Martins, 2018). 
Com relação a faixa etária, houve maior ocorrência entre indivíduos com idade entre 20 e 39 anos. Corroborando com esse achado, no estudo de Oliveira et al. (2018) essa faixa também demonstrou uma prevalência maior em comparação às outras, o que é justificado pela maior exposição à certas atividades durante essa fase da vida, como relações sexuais e utilização de drogas injetáveis.

Ainda sobre a idade, a grande quantidade de casos em adultos entre 40-59 anos demonstra que a TB afeta principalmente a população economicamente ativa (Silva, Soares, Sampaio, \& Chaves, 2017). A eficácia da vacina BCG diminui o risco dos indivíduos jovens se infectarem, entretanto a alta prevalência nos adultos pode ser associada a alguns fatores de risco, como a coinfecção de HIV/Aids. Já a incidência nos idosos está relacionada ao crescimento da expectativa de vida que facilita para que as cepas latentes obtidas no decorrer da vida se reativem de forma endógena (Barioto \& Anversa, 2015).

Quanto à raça, a mais prevalente foi a parda. Corrobora com esta afirmação o estudo realizado por Tavares et al. (2020), onde 65,20\% dos indivíduos eram da raça parda. De acordo com Pinto, Silveira, Rujula, Chiaravalloti Neto e Ribeiro (2017), a TB no Brasil associa-se principalmente aos indivíduos pardos, pretos e indígenas, pois esses grupos ainda enfrentam obstáculos discriminatórios para usar os serviços de assistência à saúde e também para ter melhores condições de renda.

No que tange à escolaridade, a maioria da população investigada tinha o ensino fundamental incompleto ou era analfabeta, resultado esse semelhante ao encontrado no estudo de Costa, Silva, Matos e Silva (2020) realizado no Piauí sobre as variáveis sociodemográficas dos casos notificados de TB, no qual o analfabetismo e a baixa escolaridade foram predominantes. Existe um estímulo cíclico em relação à baixa escolaridade e ao conhecimento da patologia, o que consequentemente reflete no não tratamento ou no abandono deste, aumentando ainda mais a transmissão e incidência da doença (Fontes et al., 2019).

A Tabela 2 representa a prevalência dos casos de TB no período analisado, pode observar que ano com maior ocorrência foi o de 2016 com 77 (23,7\%) de casos; seguido de 2018 (20,9\%); e 2019 (20,3\%) do total levantado. Houve um aumento no número de casos entre os anos de 2015 a 2016, e de 2017 a 2019 o ano de 2018 foi o mais prevalente.

Tabela 2. Prevalência dos casos de Tuberculose segundo o mês e ano de ocorrência no período de 2015 a 2019 no município de Caxias, MA. Caxias, MA, Brasil, 2020.

\begin{tabular}{|c|c|c|c|c|c|c|c|c|c|c|c|c|}
\hline \multicolumn{13}{|c|}{ Ano } \\
\hline \multirow[t]{2}{*}{ Variáveis } & \multicolumn{2}{|c|}{2015} & \multicolumn{2}{|c|}{2016} & \multicolumn{2}{|c|}{2017} & \multicolumn{2}{|c|}{2018} & \multicolumn{2}{|c|}{2019} & \multicolumn{2}{|c|}{ Total } \\
\hline & $\mathbf{N}$ & $\%$ & $\mathbf{N}$ & $\%$ & $\mathbf{N}$ & $\%$ & $\mathbf{N}$ & $\%$ & $\mathbf{N}$ & $\%$ & $\mathbf{N}$ & $\%$ \\
\hline \multicolumn{13}{|l|}{ Mês } \\
\hline Janeiro & 4 & 1,2 & 9 & 2,8 & 2 & 0,6 & 8 & 2,5 & 8 & 2,5 & 31 & 9,5 \\
\hline Fevereiro & 3 & 0,9 & 6 & 1,8 & 4 & 1,2 & 3 & 0,9 & 6 & 1,8 & 22 & 6,8 \\
\hline Março & 6 & 1,8 & 4 & 1,2 & 6 & 1,8 & 11 & 3,4 & 7 & 2,2 & 34 & 10,5 \\
\hline Abril & 7 & 2,2 & 5 & 1,5 & 11 & 3,4 & 7 & 2,2 & 7 & 2,2 & 37 & 11,4 \\
\hline Maio & 6 & 1,8 & 5 & 1,5 & 1 & 0,3 & 5 & 1,5 & 12 & 3,7 & 29 & 8,9 \\
\hline Junho & 3 & 0,9 & 6 & 1,8 & 2 & 0,6 & 6 & 1,8 & 1 & 0,3 & 18 & 5,5 \\
\hline Julho & 2 & 0,6 & 6 & 1,8 & 5 & 1,5 & 3 & 0,9 & 6 & 1,8 & 22 & 6,8 \\
\hline Agosto & 2 & 0,6 & 5 & 1,5 & 1 & 0,3 & 1 & 0,3 & 4 & 1,2 & 13 & 4,0 \\
\hline Setembro & 6 & 1,8 & 8 & 2,5 & 11 & 3,4 & 4 & 1,2 & 9 & 2,8 & 38 & 11,7 \\
\hline Outubro & 5 & 1,5 & 7 & 2,2 & 5 & 1,5 & 10 & 3,1 & 1 & 0,3 & 28 & 8,6 \\
\hline Novembro & 6 & 1,8 & 9 & 2,8 & 9 & 2,8 & 2 & 0,6 & - & - & 26 & 8,0 \\
\hline Dezembro & 3 & 0,9 & 7 & 2,2 & 4 & 1,2 & 8 & 2,5 & 5 & 1,5 & 27 & 8,3 \\
\hline Total & 53 & 16,3 & 77 & 23,7 & 61 & 18,8 & 68 & 20,9 & 66 & 20,3 & 325 & 100,0 \\
\hline
\end{tabular}

Legenda: $\mathrm{N}=$ número; $\%$ = percentual.

Fonte: Ministério da Saúde/ Sistema de Informação de Agravos de Notificação (SINAN Net-2020). 
Neste estudo a maior prevalência dos casos de TB ocorreu no ano de 2016, diferente do estudo realizado por Sousa, Sales, Gomes e Silva (2020) sobre a prevalência de TB na região nordeste no período de 2015 a 2019, no qual o maior registo aconteceu em 2018. Entretanto, ambos os estudos demonstraram uma pequena redução no ano de 2019, o que pode ser explicado pela meta do Plano Nacional de diminuir a incidência de TB para menos de 10 casos por 100.000 habitantes até 2035.

A alta dos casos em 2016 se justifica pelo maior incentivo e melhoria da Estratégia de Saúde da Família (ESF) e treinamento de profissionais de saúde, fazendo consequentemente, que os diagnósticos e tratamento se tornassem mais efetivos, melhorando a notificação dos casos (Silva et al., 2017).

De acordo com a Tabela 3, a avaliação da forma clínica da doença mostra que a pulmonar com 297 (91,4\%) casos, apresentou coeficientes mais elevados do que a extrapulmonar $(8,0 \%)$ no período analisado. Quanto ao modo entrada quase toda a totalidade $(82,8 \%)$ eram incidente, ou seja, casos novos; e apenas $22(6,8 \%)$ eram recidivos. Vale ressaltar que 63 $(19,4 \%)$ dos casos não tiveram o Tratamento Diretamente Observado (TOD), que de acordo com o Ministério da Saúde deve ser realizado, preferencialmente, em regime de TOD.

Tabela 3. Número e percentual segundo a forma clínica, tipo de entrada e a evolução dos casos de tuberculose no período de 2015-2019 no município de Caxias, MA. Caxias, MA, Brasil, 2020.

\begin{tabular}{lcccccc}
\hline & Sexo & & & & \\
\hline Variáveis & \multicolumn{3}{c}{ Masculino } & \multicolumn{2}{c}{ Feminino } & \multicolumn{2}{c}{ Total } \\
& N & \% & N & \% & N & \% \\
\hline Forma Clínica & & & & & & \\
Pulmonar & 202 & 62,2 & 95 & 29,2 & 297 & 91,4 \\
Extrapulmonar & 16 & 4,9 & 10 & 3,1 & 26 & 8,0 \\
Pulmonar + Extrapulmonar & 1 & 0,3 & - & - & 1 & 0,3 \\
Ignorado & 1 & 0,3 & - & - & 1 & 0,3 \\
Total & 220 & 67,7 & 105 & 32,3 & 325 & 100,0 \\
Tipo de Entrada & & & & & & \\
Caso novo & 186 & 57,2 & 83 & 25,5 & 269 & 82,8 \\
Recidiva & 16 & 4,9 & 6 & 1,8 & 22 & 6,8 \\
Reingresso após abandono & 11 & 3,4 & 9 & 2,8 & 20 & 6,2 \\
Transferência & 4 & 1,2 & 2 & 0,6 & 6 & 1,8 \\
Pós óbito & 3 & 0,9 & 5 & 1,5 & 8 & 2,5 \\
Total & 220 & 67,7 & 105 & 32,3 & 325 & 100,0 \\
Tratamento diretamente observado & & & & & & \\
Sim & 168 & 51,7 & 85 & 26,2 & 253 & 77,8 \\
Não & 45 & 13,8 & 18 & 5,5 & 63 & 19,4 \\
Ignorado & 7 & 2,2 & 2 & 0,6 & 9 & 2,8 \\
Total & 220 & 67,7 & 105 & 32,3 & 325 & 100,0 \\
Evolução do caso & & & & & \\
Cura & 159 & 48,9 & 75 & 23,1 & 234 & 72,0 \\
Abandono & 12 & 3,7 & 6 & 1,8 & 18 & 5,5 \\
Óbito por tuberculose & 11 & 3,4 & 8 & 2,5 & 19 & 5,8 \\
Óbito por outras causas & 24 & 7,4 & 7 & 2,2 & 31 & 9,5 \\
Transferência & 9 & 2,8 & 8 & 2,5 & 17 & 5,2 \\
Mudança de Esquema & 1 & 0,3 & - & - & 1 & 0,3 \\
Abandono Primário & 2 & 0,6 & - & - & 2 & 0,6 \\
Ignorado & 2 & 0,6 & 1 & 0,3 & 3 & 0,9 \\
Total & 220 & 67,7 & 105 & 32,3 & 325 & 100,0 \\
\hline & & & & &
\end{tabular}

Legenda: $\mathrm{N}=$ número; $\%$ = percentual.

Fonte: Ministério da Saúde/ Sistema de Informação de Agravos de Notificação (SINAN Net-2020). 
Em relação a forma clínica dados encontrados neste estudo corrobora com os resultados encontrados nos estudos de Almeida et al. (2015) ao analisarem a forma clínica de 266 casos, identificaram que 245 eram casos pulmonares, representando 92,1\% da amostra. Outros estudos também obtiveram uma proporção maior da forma clínica pulmonar do que as demais formas de TB, como nos estudos de Freitas, Santos, Silva e Rocha (2016) no município de Belém (PA) com o total de 82,5\%, Pinto et al. (2017) realizado no município de São Paulo (SP) com 75, 9\% de casos de TB pulmonar.

A prevalência da forma pulmonar pode estar relacionada principalmente na forma de transmissão, uma vez que podem ser transmitidas através da fala, espirro e tosse, o que facilita a propagação de gotículas contendo o bacilo e provocando a disseminação da doença (Bertolozzi, Takahashi, Hino, Litvoe, \& França, 2014).

Em um estudo realizado em Cajazeira-PB em que o tipo de entrada, caracterizava-se principalmente por casos novos, representando 86\% do total da amostra analisada (Almeida et al., 2015). Estes dados assim como os obtidos neste estudo não diferem das estatísticas nacionais, pois nos estudos de Fontes et al. (2019) ao analisarem o tipo de entrada da TB em todo Brasil, observou-se que a maioria dos casos, correspondendo a $81,11 \%$, são de casos novos, e a justificativa para isso estaria relacionado ao déficit de diagnóstico, ou no acompanhamento, e a eficácia do tratamento, que resultaria na diminuição da incidência de casos recidivos.

Relacionado ao TDO é importante ressaltar que é uma estratégia essencial no enfrentamento da TB, em locais que utilizam o TDO observa-se a diminuição da ocorrência de casos recidivos, apesar que possa acontecer o abandono do tratamento, a estratégia do TOD viabiliza maiores chances de resolutividade da TB (Junges, Burille, \& Tedesco, 2020).

A TB é uma doença é curável, o tratamento permite a cura dos casos novos, pois estes são sensíveis aos medicamentos antiTB, porém é necessário seguir toda a terapêutica adequadamente, respeitando as doses e o tempo (Brasil, 2011). Mas uma abordagem humanizada é imprescindível para o estabelecimento de vínculo entre profissional de saúde e usuário, proporcionar a escuta ao paciente em relação a dúvidas, e angústias, possibilita que o profissional identifique vulnerabilidades, e auxilie no diagnóstico e na adesão ao tratamento (Brasil, 2019).

Rabahi, Silva Júnior, Ferreira, Tannus-Silva e Conde (2017) destacam em seus estudos que a eficácia do esquema terapêutico antituberculose é de até $95 \%$, porém a efetividade do tratamento varia muito de acordo com o local, estando a média nacional em torno de 70\%. O abandono do tratamento da TB representa um problema de saúde pública, pois possibilita o aumento de contagio, além de altos custos no tratamento, resistência aos fármacos e morbimortalidade (Silva, Moura, \& Caldas, 2014).

Com relação a forma clinica extrapulmonar observada em 27 (8,3\%) dos casos a ganglionar periférica foi a mais prevalente com nove (33\%) dos casos, seguido da pleural (30\%) e miliar (15\%), conforme demonstrado na Figura 1. 
Figura 1. Distribuição da forma clínica extrapulmonar dos casos de tuberculose no período de 2015-2019 no município de Caxias, MA. Caxias, MA, Brasil, 2020.

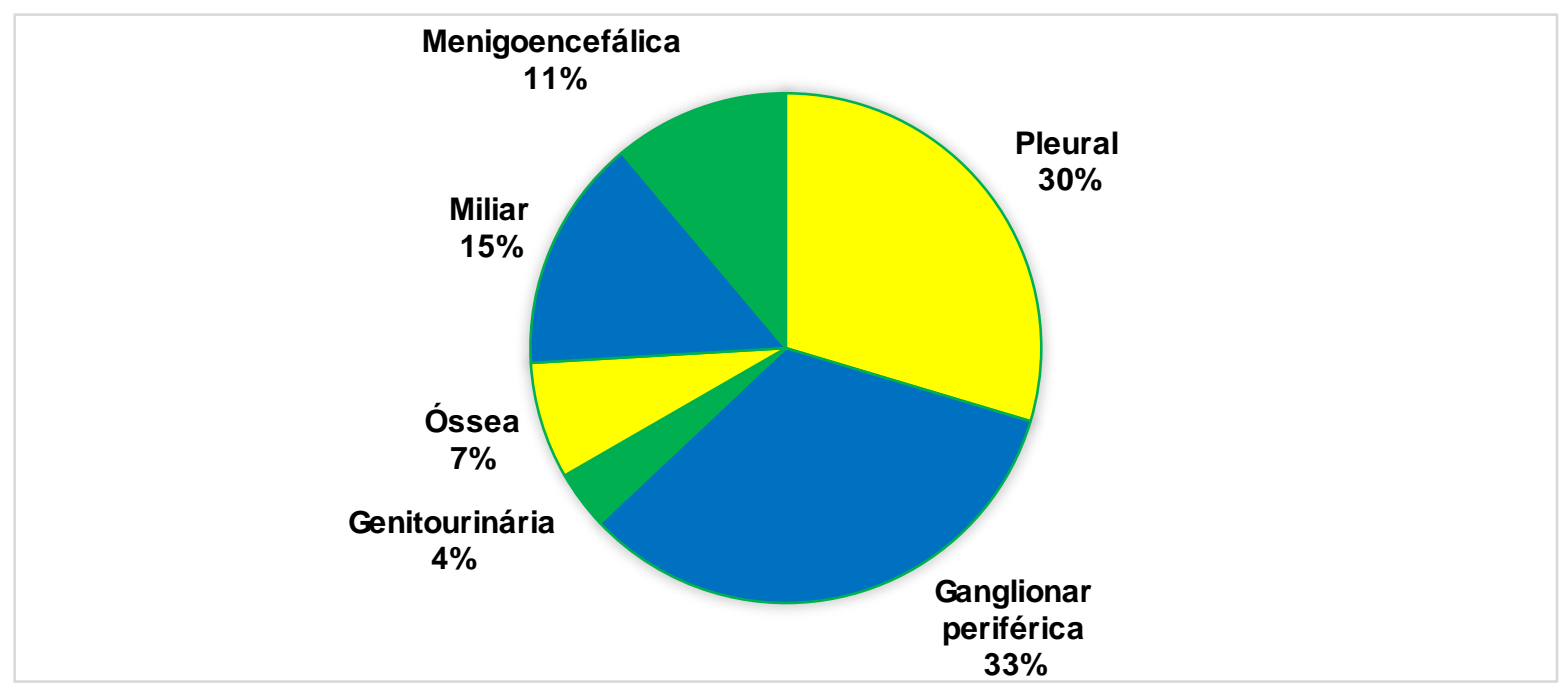

Legenda: $\mathrm{N}=$ número; $\%$ = percentual.

Fonte: Ministério da Saúde/ Sistema de Informação de Agravos de Notificação (SINAN Net), 2020.

A TB extrapulmonar apresenta uma reduzida taxa de incidência nos últimos anos, porém está forma clínica ainda representa um grande agravo em saúde pública, tendo um difícil diagnostico uma vez que pode apresentar uma variedade de casos clínicos e muitas vezes o despreparo dos profissionais em relação a doença pode comprometer na prestação de cuidados (Barros, Pinto, Silva, Silva, \& Figueiredo, 2014).

Dados desta pesquisa corrobora com os estudos de Almeida et al. (2015) na qual dentre as formas de TB extrapulmonar as de maior incidência foram a Ganglionar periférica em que no estudo apresentou-se com 13 casos, tendo um percentual de $61,9 \%$, e a forma Pleural com cinco casos, e percentual de $23,8 \%$.

Os sinais e sintomas da TB extrapulmonar dependem dos sistemas ou órgãos acometidos. Sendo os casos de TB ganglionar e pleural as mais incidentes das tuberculoses extrapulmonar, em pacientes coinfectados pelo HIV, e com imunocomprometimento grave são as mais vulneráveis a essa patologia (Brasil, 2019).

A Figura 2 mostra o percentual de doenças e agravos associados dos casos de TB, no qual os registros que apresentaram maior índice foram tabagismo com 77 (23,7\%) dos casos, seguido de alcoolismo (19,7\%) e diabetes (16\%), respectivamente. Ainda de acordo com a Figura 2 pode-se observar que uma minoria apresentava HIV (5,5\%) e AIDS (4,6\%). 
Figura 2. Percentual de doenças e agravos associados dos casos de tuberculose no período de 2015-2019 no município de Caxias, MA. Caxias, MA, Brasil, 2020.

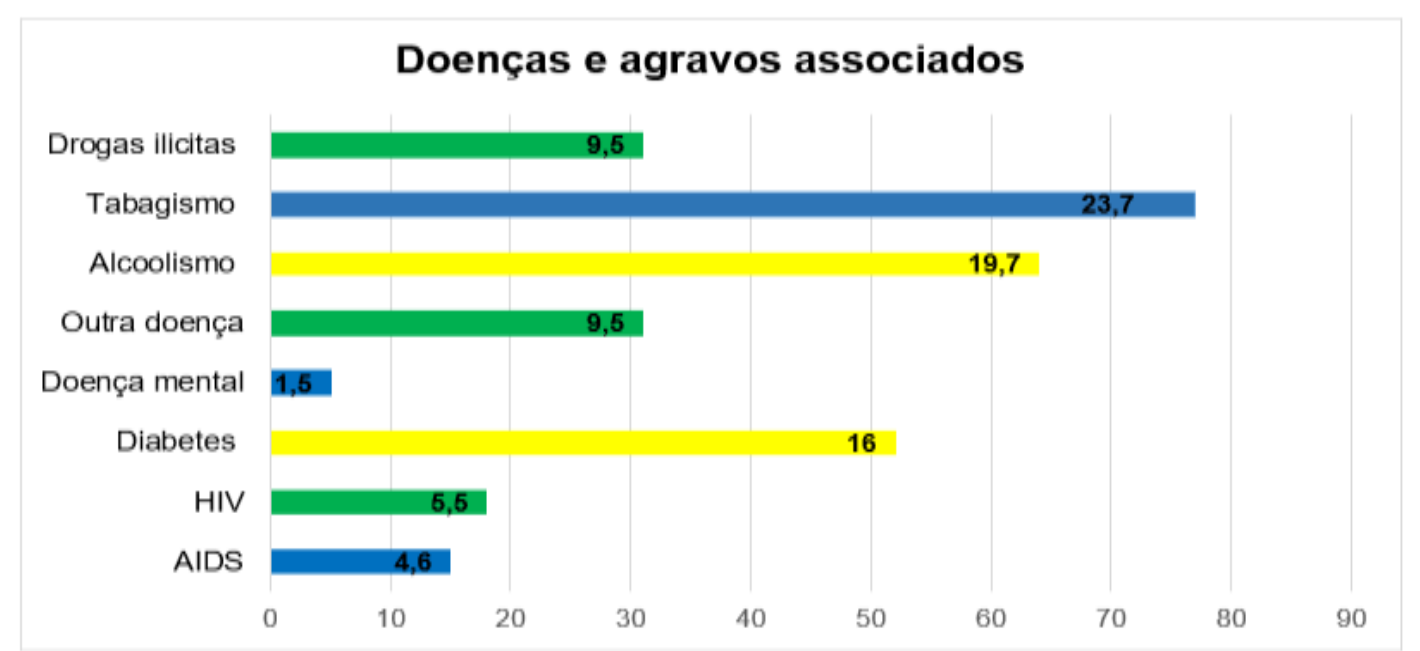

Fonte: Ministério da Saúde/ Sistema de Informação de Agravos de Notificação (SINAN Net), 2020.

A TB é uma doença negligenciada que se relaciona a diversos fatores que podem contribuir de forma negativa para o quadro clínico do paciente, dentre eles, se destaca o tabagismo. Onde o mesmo desempenha o encargo de aumentar a taxa de mortalidade da TB, ou até mesmo aumentando as chances de o indivíduo contrair a infecção (Silva et al., 2018). Tendo em vista que o hábito de fumar tem relação direta à reincidência dos casos de TB (Fregona et al., 2017).

A incidência da doença também esteve estritamente associada ao alcoolismo, onde um estudo realizado por Deus Filho e Carvalho (2018) mostrou que 30\% dos pacientes com TB ativa eram consumidores frequentes de álcool. Segundo Soares et al. (2020), o alcoolismo se destaca tanto por piorar o quadro clínico dos pacientes quanto por ser um fator de risco para o surgimento da patologia.

Observou-se ainda que existe um nexo que liga a TB ao HIV/AIDS, onde encontrou-se uma prevalência significativa de infecção por HIV entre os pacientes que apresentavam TB (Chaves, Carneiro, Santos, Sarges, \& Neves, 2017). É evidenciado que devido a imunidade baixa, as pessoas que apresentam HIV/AIDS podem adoecer gravemente ou até mesmo morrer caso não consigam ter um tratamento adequado e eficaz para a TB (Magno et al., 2017).

Segundo Novotny, Hendrickson, Soares, Sereno e Kiene (2017) a TB, o tabagismo e o HIV/AIDS apesar de agirem separadas, muitas vezes formam uma sindemia, ou seja, atuam juntas em uma espécie de trabalho conjunto para enfraquecer o sujeito. Neste ponto, abordagens de tratamentos tradicionais ou separados não são capazes de solucionar os problemas, sendo necessários programas que englobem tanto a doença quando os fatores de risco e patologias adjacentes.

Outro fator que influencia diretamente a doença ou o seu aparecimento é a Diabetes Mellitus (DM), onde pessoas que já tem a comorbidades apresentam mais chances de desenvolverem um quadro de TB no futuro (Soares et al., 2020). Não existe um consenso na literatura, porém, é sabido que a DM pode piorar os sintomas apresentados ou até mesmo se ligar a casos de recidivas de TB (Abreu, Rolim, Sousa, \& Oliveira, 2020).

\section{Considerações Finais}

Este estudo permitiu observar os aspectos epidemiológicos da TB no município de Caxias, do Estado do Maranhão. Onde se evidenciou a prevalência de casos em pessoas do sexo masculino que se enquadravam na faixa etária de 20 a 59 anos e de etnia parda, entretanto, destaca-se ainda que, os idosos com idade entre 60 anos ou mais foram bastante acometidos pela 
doença, mostrando que o agravo está presente em qualquer faixa etária. Afirmando seu perfil de doença negligenciada, manifestou-se principalmente em pessoas analfabetas ou com ensino fundamental incompleto.

Diante dos problemas encontrados torna-se claro que a TB continua sendo um grande problema de saúde pública tanto no Brasil como em alguns outros países ao redor do planeta, sobretudo em países de terceiro mundo. É necessário que mais ações voltadas ao combate da patologia sejam colocadas em prática e que os governos enxerguem a extensão do problema.

Nesse aspecto, a enfermagem exerce um importante papel, estando na linha de frente na prestação de cuidados aos pacientes acometidos pela patologia. Sendo importância avaliar o grau de conhecimento dos clientes para assegurar que o tratamento seja efetivo e evitar a reincidência dos casos, a busca ativa é umas das formas de descobrir novos casos e, realizar o atendimento o mais rapidamente possível, evitando a transmissão da doença e o agravamento do quadro clínico do indivíduo. A educação em saúde também se faz presente como forma de prevenir o surgimento da patologia e garantir que a população saiba identificar sinais e sintomas.

Como limitações do estudo tem-se o fato de que algumas vezes, os dados apresentados no sistema podem não ser totalmente fidedignos ao número de casos reais, ou ainda, no momento de inserir os dados, os profissionais podem deixar de cadastrar informações que possivelmente seriam úteis a estudos posteriores.

Por fim, a pesquisa possibilitou expandir os conhecimentos acerca da TB e seus aspectos epidemiológicos. Considerase que mais estudos devem ser realizados nessa linha de investigação, a fim de provar a necessidade de buscar novas formas de abordar a temática, visando diminuir o número de casos em todo o mundo ou até mesmo, buscar a erradicação total da doença. Vale destacar ainda, a importância do cuidado multidisciplinar no tratamento dos pacientes acometidos pela patologia.

\section{Referências}

Abreu, R. G., Rolim, L. S., Sousa, A. I. A., \& Oliveira, M. R. F. (2020). Tuberculose e diabetes: associação com características sociodemográficas e de diagnóstico e tratamento. Brasil, 2007-2011. Rev. bras. Epidemiol, 23, 01-13.

Almeida, A. A., Barros, H. J. M., Silva, T. C., Medeiros, R. L. S. F. M., Nascimento, M. M. P., \& Temoteo, R. C. A. (2015). Perfil clínico-epidemiológico de casos de tuberculose. Rev enferm UFPE on line, 9(9), 1007-1017.

Barioto, J. G., \& Anversa, L. (2015). Perfil epidemiológico dos casos de tuberculose notificados no município de Bauru, estado de São Paulo, Brasil. BEPA, 12(134), 01-11.

Barros, P. G., Pinto, M. L., Silva, T. C., Silva, E. L., \& Figueiredo, T. M. R. M. (2014). Perfil Epidemiológico dos casos de Tuberculose Extrapulmonar em um município do estado da Paraíba, 2001-2010. Cad. Saúde Colet., 22(4), 343-350.

Bertolozzi, M. R., Takahashi, R. F., Hino, P., Litvoc, M., \& França, F. O. S. (2014). O controle da tuberculose: um desafio para a saúde pública. Rev Med, 93(2), 83-89.

Brasil. (2019). Manual de Recomendações para o Controle da Tuberculose no Brasil. Brasília: Ministério da Saúde.

Brasil. (2011). Tratamento diretamente observado (TDO) da tuberculose na atenção básica: protocolo de enfermagem. Brasília: Ministério da Saúde.

Carvalho, A. C. C., Cardoso, C. A. A., Martire, T. M., Migliori, G. B., \& Sant'Anna, C. C. (2018). Aspectos epidemiológicos, manifestações clínicas e prevenção da tuberculose pediátrica sob a perspectiva da Estratégia End TB. J. bras. pneumol, (44)2, 134-144.

Carvalho, F. A. F. T. (2019). Análise da distribuição espacial dos casos de tuberculose pulmonar na área insular do município de Santos/SP e a estratégia DOTS, 2006 - 2014. (Tese de Doutorado) Programa de Pós-Graduação stricto sensu em Saúde Coletiva, Universidade Católica de Santos, Santos, SP, Brasil.

Carvalho, L. O. R., Duarte, F. R., Menezes, A. H. N., \& Souza, T. E. S. (2019). Metodologia científica: teoria e aplicação na educação a distância: PetrolinaPE: Fundação Universidade Federal do Vale do São Francisco.

Chaves, E. C., Carneiro, I. C. R. S., Santos, M. I. P. O., Sarges, N. A., \& Neves, E. O. S. (2017). Aspectos epidemiológicos, clínicos e evolutivos da tuberculose em idosos de um hospital universitário em Belém, Pará. Rev. bras. geriatr. Gerontol, 20(1), 45-55.

Costa, R. H. F., Silva, H. R., Matos, R. P. S., \& Silva, Y. A. (2020). Estudo epidemiológico das variáveis sociodemográficas dos casos notificados de tuberculose no estado do Piauí. Research, Society and Development, 9(4), 1-11.

Deus Filho, A., \& Carvalho, I. M. S. (2018). Epidemiological profile of patients with tuberculosis in a teresina-pi university hospital. Jornal de Ciências da Saúde do Hospital Universitário da UFPI, 1(1), 51-60.

Ferreira Neto, P. T. P., Oliveira, V. G., \& Pimenta, F. P. (2020). Novas tecnologias para o tratamento da tuberculose: o que as patentes nos dizem. Quím. Nova, 43(7), 998-1009. 
Fontes, G. J. F., Silva, T. G., Sousa, J. C. M., Feitosa, A. N. A., Silva, M. L., Bezerra, A. L. D., \& Assis, E. V. (2019). Perfil epidemiológico da tuberculose no Brasil no período de 2012 a 2016. Rev. Bra. Edu. Saúde, 9(1), 19-26.

Fregona, G., Cosme, L. B., Moreira, C. M. M., Bussular, J. L., Dettoni, V. V., Dalcolmo, M. P., \& Maciel, E. L. N. (2017). Fatores associados à tuberculose resistente no Espírito Santo, Brasil. Rev. Saúde Pública, 51(41), 1-11.

Freitas, W. M. T. M., Santos, C. C., Silva, M. M., \& Rocha, G. A. (2016). Perfil clínico-epidemiológico de pacientes portadores de tuberculose atendidos em uma unidade municipal de saúde de Belém, Estado do Pará, Brasil. Revista Pan-Amazônica de saúde, 7(2).

Junges, J. R., Burille, A., \& Tedesco, J. (2020). Tratamento Diretamente Observado da tuberculose: análise crítica da descentralização. Interface (Botucatu), $24,1-13$.

Magno, E. S., Saraceni, V., Souza, A. B., Magno, R. S., Saraiva, M. G. G., \& Bührer-Sékula, S. (2017). Fatores associados à coinfecção tuberculose e HIV: o que apontam os dados de notificação do Estado do Amazonas, Brasil, 2001-2012. Cad. Saúde Pública, 33(5), 1-11.

Novotny, T., Hendrickson, E., Soares, E. C. C., Sereno, A. B., \& Kiene, S. M. (2017). HIV/AIDS, tuberculose e tabagismo no Brasil: uma sindemia que exige intervenções integradas. Cad. Saúde Pública, 33(3), 1-4.

Oliveira, L. B., Costa, C. R. B., Queiroz, A. A. F. L. N., Araújo, T. M. E., Sousa, K. A. A., \& Reis, R. K. (2018). Análise epidemiológica da coinfecção tuberculose/HIV. Cogitare Enferm, 23(1), 1-8.

Pereira, A. S., Shitsuka, D. M., Parreira, F. J., \& Shitsuka, R. (2018). Metodologia da pesquisa científica. UFSM.

Pinto, P. F. P. S., Silveira, C., Rujula, M. J. P., Chiaravalloti Neto, F., \& Ribeiro, M. C. S. A. (2017). Perfil epidemiológico da tuberculose no município de São Paulo de 2006 a 2013. Rev Bras Epidemiol, 20(3), 549-557.

Rabahi, M. F., Silva Júnior, J. L. R., Ferreira, A. C. G., Tannus-Silva, D. G. S., \& Conde, M. B. (2017). Tratamento da tuberculose. J Bras Pneumol, 43(5), 472-486.

Santana, N. A., Fraga, I. M. N., \& Moreira, T. (2019, maio). Adesão ao tratamento da tuberculose e a contribuição do enfermeiro em Sergipe. Anais do Congresso Internacional de Enfermagem, Aracaju, SE, Brasil, 2.

Santos, T. A., \& Martins, M. M. F. (2018). Perfil dos casos de reingresso após abandono do tratamento da tuberculose em Salvador, Bahia, Brasil. Cad. saúde colet., 26(3), 233-240.

Silva, D. R., Muñoz-Torrico, M., Duarte, R., Galvão, T., Bonini, E. H., Arbex, F. F., \& Mello, F. C. Q. (2018). Fatores de risco para tuberculose: diabetes, tabagismo, álcool e uso de outras drogas. J. bras. pneumol, 44(2), 145-152.

Silva, P. F., Moura, G. S., \& Caldas, A. J. M. (2014). Fatores associados ao abandono do tratamento da tuberculose pulmonar no Maranhão, Brasil, no período de 2001 a 2010. Cad. Saúde Pública, 30(8), 1745-1754.

Silva, W. A., Soares, Y. J. A., Sampaio, J. P. S., \& Chaves, T. V. S. (2017). Perfil epidemiológico dos casos notificados de tuberculose no Piauí nos anos de 2010 a 2014. R. Interd., 10(3), 31-38.

Soares, K. R. M., Nunes, E. M., Leite, K. N. Z, Soares, C. M., \& Medeiros, H. R. L. (2019). Perfil epidemiológico da tuberculose no município de Patos- PB no período de 2013 a 2017. Temas em Saúde, 19(3), 460-473.

Soares, V. M., Almeida, I. N., Figueredo, L. J. A., Haddad, J. P. A., Oliveira, C. S. F., Carvalho, W. S., \& Miranda, S. S. (2020). Fatores associados à tuberculose e à tuberculose multirresistente em pacientes atendidos em um hospital de referência terciária em Minas Gerais, Brasil. J. bras. pneumol, 46(2), 1-

Sousa, G. O., Sales, B. N., Gomes, J. G. F., \& Silva, M A. (2020). Epidemiologia da tuberculose no nordeste do Brasil, 2015 - 2019. Research, Society and Development, $9(8), 1-12$.

Tavares, C. M., Cunha, A. M. S., Gomes, N. M. C., Lima, A. B. A., Santos, I. M. R., Acácio, M. S., \& Souza, C. D. F. (2020). Tendência e caracterização epidemiológica da tuberculose em Alagoas, 2007-2016. Cad. Saúde Colet., 28(1), 107-115. 